\title{
Preliminary Normative Study of ImPACT® in Finnish Professional Male Ice Hockey Players
}

\section{Vartiainen, Matti V}

2021-01-01

Vartiainen , M V , Peltonen , K , Holm , A , Koskinen , S , Iverson , G L \& Hokkanen , L 2021

, ' Preliminary Normative Study of ImPACT® in Finnish Professional Male Ice Hockey

Players ' , Applied neuropsychology-Adult , vol. 28 , no. 1 , pp. 53-59 . https://doi.org/10.1080/23279095.2019.15921

http://hdl.handle.net/10138/328412

https://doi.org/10.1080/23279095.2019.1592175

acceptedVersion

Downloaded from Helda, University of Helsinki institutional repository.

This is an electronic reprint of the original article.

This reprint may differ from the original in pagination and typographic detail.

Please cite the original version. 
A Preliminary Normative Study of ImPACT® in Finnish Professional Male Ice Hockey Players

Matti V. Vartiainen, M.Sc.

Department of Psychology and Logopedics, Faculty of Medicine, University of Helsinki, Helsinki, Finland

Email: m.vartiainen@kolumbus.fi

Kati Peltonen, M.A., M.S.Ed.

Department of Psychology and Logopedics, Faculty of Medicine,

University of Helsinki, Helsinki, Finland

kati.peltonen@helsinki.fi

Anu Holm, Ph.D.

Department of Clinical Neurophysiology, Satakunta Central Hospital, Pori, Finland Email: anu.holm@satadiag.fi

Sanna Koskinen, Ph.D

Department of Psychology and Logopedics, Faculty of Medicine, University of Helsinki, Helsinki, Finland sanna.koskinen@helsinki.fi

Grant L. Iverson, Ph.D.

Department of Physical Medicine and Rehabilitation, Harvard Medical School; MassGeneral Hospital for Children ${ }^{\mathrm{TM}}$ Sport Concussion Program; Spaulding Rehabilitation Hospital; \& Home Base, A Red Sox Foundation and Massachusetts General Hospital Program, Boston, Massachusetts, USA Email: giverson@mgh.harvard.edu

Laura Hokkanen, Ph.D

Department of Psychology and Logopedics, Faculty of Medicine, University of Helsinki, Helsinki, Finland laura.hokkanen@helsinki.fi

Corresponding author: Matti V Vartiainen, phone: +35850 5853884

Email: m.vartiainen@kolumbus.fi

University of Helsinki, Finland

P.O.Box 21

00014 HY, Helsinki, Finland

\section{Running title: ImPACT in Finnish Ice Hockey}




\title{
A Preliminary Normative Study of ImPACT® in Finnish Professional Male Ice Hockey Players
}

\author{
Abstract \\ Assessment of cognition is an important part of concussion management. The \\ common paradigm of baseline and post-injury evaluations is recommended but \\ due to the often lacking baseline data, reliable normative values are needed. The \\ Immediate Post-Concussion Assessment and Cognitive Testing (ImPACT®) \\ battery is a widely used method of cognitive assessment offering several language \\ options. There are few comparative studies between language groups on this test \\ battery. ImPACT was administered at baseline to 184 Finnish male Professional \\ Ice Hockey league players. The performance of the Finnish sample differed from \\ previously published English and Czech language samples on the Visual Motor \\ Speed and Reaction Time composites with medium effect-sizes $(d=0.38-0.52)$. \\ Age, but not education or prior concussions, was associated with ImPACT \\ performance. ImPACT performance is not uniform across language and culture \\ groups and the findings highlight the need for language-specific norms. Finnish \\ reference values for ImPACT for ages $<20,20-26$, and $>26$ are presented. A \\ similar approach is encouraged for other languages.
}

Key words: Sport concussion, Cognition, Baseline, Norms, Cultural differences 


\section{INTRODUCTION}

Approximately 1.6 to 3.8 million sport related concussions (SRC) occur annually in United States (Daneshvar, Nowinski, McKee, \& Cantu, 2011; Langlois, Rutland-Brown, \& Wald, 2006). In ice hockey the risk of injury is higher than in many other sports: the total rate of concussions among 15 different collegiate sports was estimated at 0.28 per 1000 athletic-exposures but in male ice hockey the rate was 0.41 (Daneshvar et al., 2011; Hootman, Dick, \& Agel, 2007). Since the first International Conference on Concussion in Sport in Vienna 2001, neuropsychological testing has played an important role in SRC evaluation and management (Aubry et al., 2002; McCrory et al., 2017). As a part of a multidomain assessment protocol, neuropsychological testing offers performancebased information that aids in the clinical management of SRC (Broglio, Macciocchi, \& Ferrara, 2007; Echemendia et al. 2013; Grindel, Lovell, \& Collins, 2001; Putukian, 2011). Several computerized tests are available, and the reliability of the method needs to be taken into consideration (Farnsworth, Dargo, Ragan, \& Kang, 2017). Accurate diagnosis and management might reduce the risk of repeated injury (McCrea et al., 2009) and prolonged recovery (Asken et al., 2016; Elbin et al., 2016).

The Immediate Post-Concussion Assessment and Cognitive Testing (ImPACT®) is a widely used computerized test battery considered to be useful in screening cognitive changes related to SRC (Broglio et al., 2007; Elbin, Schatz, \& Covassin, 2011; Fazio, Lovell, Pardini, \& Collins, 2007; Iverson, Lovell, \& Collins, 2003; Lovell \& Collins, 2002; Schatz, Pardini, Lovell, Collins, \& Podell, 2006). Similar to other computerized tests, concerns regarding a less-than desirable reliability have been raised for ImPACT (Farnsworth et al. 2017). The 
test battery consists of three main parts: demographic data, health history, and prior history of concussions; the Post-Concussion Scale for measuring subjective symptoms; and a computerized neurocognitive test. Iverson, Lovell, and Collins (2003) have defined reference values for reliable change for ImPACT neurocognitive test scores that can be used when baseline scores are available for individual comparison after concussion. If baseline scores are not available, postinjury scores have typically been compared to ImPACT normative data, collected in the United States, stratified based on age and gender. Using normative data for interpretation may be beneficial even if baseline comparisons are available (Schatz \& Robertshaw, 2014; Schmidt, Register-Mihalik, Mihalik, Kerr, \& Guskiewicz, 2012).

Although ImPACT has been translated into many languages, there are limited studies that assess the adequacy of these translations or the cultural equivalence of each test version. Cultural, ethnic, and linguistic differences on traditional paper-and-pencil neuropsychological tests are widely recognized (Agranovich \& Puente, 2007; Bure-Reyes et al., 2013; Cherner et al., 2007; Norman et al., 2011; Shuttleworth-Edwards et al., 2004). It is not known whether ImPACT norms developed in the United States are an appropriate reference base for the population in other countries. Differences in baseline performance between English speaking and Spanish speaking Hispanic youth athletes have been found on ImPACT (Ott, Schatz, Solomon, \& Ryan, 2014). Significant variability has also been observed in test-retest reliabilities among different linguistic subsamples in National Hockey League athletes in the USA (Bruce, Echemendia, Meeuwisse, Comper, \& Sisco, 2014). Results from French-, Czech-, and Swedish-speaking adult subsamples were compared by Bruce et al. (2014) 
and some performance differences were noted. The primary aims of this study are (a) to compare the baseline cognitive performance of Finnish professional male ice hockey players to other published language groups, and (b) to study the associations between age, education, and prior self-reported concussions and cognitive test performance in this adult sample. A secondary aim is to establish Finnish normative reference values for the ImPACT test scores.

\section{METHODS}

Participants. A total of 185 professional male ice hockey players from five teams in the Finnish national league were tested to obtain pre-season baseline data [seasons: 2012-13 $(n=118)$ and 2013-14 $(n=67)]$. The results of the first baseline only were included for those participating both times. One player was excluded from the study because of invalid test results, defined as an ImPACT Impulse Control Composite score above 30. The study was approved by the Ethical Committee of the Helsinki Uusimaa Hospital District, and each participating subject signed a written informed consent according to the Declaration of Helsinki.

ImPACT. Cognitive performance was assessed with Finnish language version of ImPACT. The six cognitive subtests included are Word Memory, Design Memory, X's and O's, Symbol Match, Color Match, and Three Letter Memory. Composite index scores for Verbal Memory, Visual Memory, Visual Motor Speed, Reaction Time, and Impulse Control are derived by combining correct responses and reaction time measures across subtests. Impulse Control is a measure for errors on testing and used for assessing the test validity. The test was conducted in a quiet environment, at least 15 minutes after exercise (8am to 4pm) by two experienced health care professionals (authors MV and KP). The study 
protocol was described previously (Vartiainen et al., 2015). Years of education, special education needs, and the number of prior self-reported concussions were collected at the time of testing. Special education needs included having a diagnosed learning disability or ADHD, or having attended special education classes or speech therapy.

Statistical Analyses. The means ( $M)$, medians $(M d)$, standard deviations $(S D)$, and ranges are presented as descriptive statistics. Composite scores for Verbal Memory, Visual Memory, Visual Motor Speed, and Reaction Time were normally or near normally distributed when examined using the Shapiro-Wilk test and Normal Q-Q plots. The Impulse Control composite score was skewed and is analyzed separately using non-parametric statistics. The performance of the Finnish players was compared with published adult values of English-, French-, Czech-, and Swedish-speaking subsamples from Bruce et al. (2014) using GraphPad QuickCalcs online calculator with two-tailed $t$-tests. Adjusting for the multiple comparisons yielded a required Bonferroni corrected $p$-value of 0.003 . For the pairwise group comparisons, the effect size $d$ corrected for different group sizes and standard deviations is reported. Effect sizes around 0.2 are considered small, those around 0.5 medium, and those around 0.8 large (Cohen, 1988).

The effect of age in the Finnish sample was studied by dividing the participants into three groups: (i) $<20$ years $(n=53)$, (ii) $20-26$ years $(n=72)$, and (iii) $>26$ years $(n=59)$. The effect of education was studied by forming two groups: (i) 12 years or more (corresponds to completed high school or more, $n=70$ ), and (ii) less than 12 years of education $(n=102)$. Twelve participants had missing information on education. The effect of prior concussions was studied by 
forming two groups based on reported history: (i) players with no prior concussions ( $n=99)$, and (ii) players with one or more prior concussions $(n=85)$.

General linear models (GLM) were used to assess the effects of demographic and injury history variables on the four composite scores. A oneway between subjects multivariate analysis of variance (MANOVA with subsequent ANOVAs and Bonferroni corrected pair-wise comparisons) was first carried out to assess the impact of age on the ImPACT composite scores (Verbal Memory, Visual Memory, Visual Motor Speed, and Reaction Time). Partial eta squared $\left(\eta_{p}^{2}\right)$ values are reported for effect sizes. Effect sizes around 0.01 are considered small, those around 0.06 medium, and those above 0.14 large (Cohen, 1988). The effects of education and concussion history were examined with twoway MANOVAs also including age as a factor. Impulse Control was analyzed separately with Mann-Whitney U and Kruskal-Wallis tests. IBM SPSS Statistics 25.0 (IBM Corp. Armonk, NY, USA) was used to perform the analyses.

\section{RESULTS}

The mean age of the 184 participants was 23.8 years $(S D=5.6$, range $=16$ 40). All players were Finnish and Caucasian. Mean education was 11.58 years $(S D=1.4$, range $=9-16)$ and the mean number of prior concussions sustained was $0.90(M d=0, S D=1.2$, range $=0-7)$.

Statistically significant differences in ImPACT test performances between language groups emerged on the Visual Motor Speed and Reaction Time composites (Table 1). Compared to the English-speaking subsamples, Finnish participants had lower scores on Visual Motor Speed $[t(301)=3.23, p=.001$, $|d|=0.38]$ and slower Reaction Times $[t(301)=4.11, p<.001,|d|=0.48]$. Compared to the Czech speaking subsample, Finnish participants had higher scores on 
Visual Motor Speed $[t(224)=3.12, p=.002,|d|=0.52]$. There were no differences in age or education between the groups.

Within the Finnish sample, there was a statistically significant difference in ImPACT test performance between the three age groups $[F(8,356)=2.69$, $p=.007$; Wilks' Lambda $\left.=0.89, \eta_{\mathrm{p}}{ }^{2}=0.06\right]$. Differences associated with age occurred on Visual Motor Speed $\left[F(2,181)=3.24, p=.041 ; \eta_{\mathrm{p}}{ }^{2}=0.04\right]$ and Reaction Time $\left[F(2,181)=9.21 p<.001 ; \eta_{\mathrm{p}}{ }^{2}=0.09\right]$ composites. The oldest group $(>26$ years olds) had lower scores than the middle age group (20-26 years olds) on the Visual Motor Speed composite ( $p=.037)$. For the Reaction Time composite, the oldest group was slower than the youngest group $(\mathrm{p}=0.001)$ and the middle group $(p=0.001)$.

The three age groups differed in level of education and concussion history, but not in their special education histories (Table 2). Level of education was not associated with cognitive test performance $[F(4,163)=0.99, p=.42$; Wilks' Lambda $\left.=.98, \eta_{\mathrm{p}}{ }^{2}=0.02\right]$, nor was there an interaction between age and education $\left[F(8,326)=0.74, p=.66\right.$; Wilks' Lambda $\left.=.97, \eta_{\mathrm{p}}{ }^{2}=0.02\right]$ while the effect of age remained significant $[F(8,326)=2.04, p=.041$; Wilks' Lambda $=0.91$, $\left.\eta_{\mathrm{p}}{ }^{2}=0.05\right]$. There was also no statistically significant difference between the previously concussed and non-concussed group $[F(4,175)=0.036, p=.99$; Wilks Lambda $\left.=1.00, \eta_{\mathrm{p}}{ }^{2}=0.001\right]$ or an interaction between age and concussion history $\left[F(8,350)=0.79, p=.61 ;\right.$ Wilks' Lambda $\left.=.97, \eta_{\mathrm{p}}{ }^{2}=0.02\right]$ while the effect of age remained significant $\left[F(8,350)=2.47, p=.013\right.$; Wilks' Lambda $\left.=0.90, \eta_{\mathrm{p}}{ }^{2}=0.05\right]$. Impulse Control was associated with age $[\mathrm{K}-\mathrm{W}(2)=26.04, \mathrm{p}<0.001]$ but not with education level $(\mathrm{p}=.09)$ or with concussion history $(\mathrm{p}=.25)$. ImPACT normative values for each age group are presented separately in Table 3. 


\section{DISCUSSION}

The performance of Finnish professional male ice hockey players aged 16 to 40 years on the ImPACT test differed from the previously published English and Czech language samples (Bruce et al., 2014) on the Visual Motor Speed and Reaction Time composites. In the Finnish sample, performance on ImPACT was associated with age, but not education or prior concussions. Finnish normative baseline values for ImPACT tests were calculated for the three age groups.

The several available language options within ImPACT make the test suitable in the multilingual world of sport. The test can be used both in different countries and in any one country in teams with athletes speaking different languages. There have been concerns, however, that norms developed in the US may not be a suitable reference base for different ethnic populations (Bruce et al., 2014; Ott et al., 2014; Shuttleworth-Edwards, Whitefield-Alexander, Radloff, Taylor, \& Lovell, 2009; Tsushima \& Siu, 2014). Studies have suggested that US normative data on ImPACT are appropriate for use with South African athletes (Shuttleworth-Edwards et al., 2009) as well as Hawaiian high school athletes (Tsushima \& Siu, 2014) whose first language is English. Significant variability in test-retest reliability as well as performance has been found, however, among the different language versions of ImPACT (Bruce et al., 2014; Echemendia et al., 2016; Ott et al., 2014). In the present study, the Finnish sample differed from the published mean values for English- and Czech speaking subsamples (Bruce et al., 2014). The effect size, while suggesting considerable overlap of groups (Zakzanis, 2001), was medium (Cohen, 1988). Based on these comparisons, having language-specific normative data might improve clinical interpretations of cognitive test scores derived from ImPACT. 
ImPACT scores differed by age in our sample. There was no association between education and cognitive performance, after controlling for age, possibly due to the fairly small amount of educational variation in this sample of professional athletes. In Finland the compulsory education is 9 years, completed typically at the age of 16 . Twelve years include additional three years of high school. Levels beyond that are related to college or higher education. In addition to years of education, other achievement related variables such as learning difficulties and ADHD are worth considering in SRC management. They were evenly distributed across the three age groups in our sample and were not included in the linear models. Some studies have suggested separate reference values for this special group for pen-and-pencil tests (Collins et al., 1999; Merrit et al., 2017) as well as for ImPACT (Elbin et al., 2013; Peltonen et al., 2018; Zuckerman, Lee, Odom, Solomon, \& Sills, 2013). Especially in youth athletes, learning difficulties may indicate different maturational paths compared to typical learners (Peltonen et al., 2018). In adults this is less studied, and the current sample was too small for stratification. Performance on pre-season ImPACT in the present study was not different in those with or without a history of concussion. This in line with several previous studies suggesting no differences in cognitive test performance associated with prior concussions (Brooks et al., 2013; Brooks et al., 2016; Brooks et al., 2018; Iverson, Brooks, Lovell, \& Collins, 2006; McKay et al., 2014).

This study has several limitations. The different language groups were not assessed as part of the present study; the data for comparisons came from a published article. Despite the same age range, education and elite level, and described similar testing conditions, there may be other factors influencing the 
comparability of the findings. Furthermore, the current results pertain to professional male ice hockey players and may therefore not be generalizable to other sports, to non-athletes, or to women. Participating teams came from Southern parts of Finland, near the capital region, which may also limit generalizability. The group sizes, although larger than those of Bruce et al. (2014) were small when subdivided into age groups. Therefore, the normative values obtained should be considered preliminary.

In conclusion, the cognitive test results in this study, derived from ImPACT, differed from those previously published from elite English and Czech speaking hockey players, justifying separate norms for athletes from diverse cultural and language backgrounds. This study presents preliminary normative data for professional Finnish ice hockey players that can be used to interpret cognitive test performance following a SRC especially when baseline data is not available for comparison. Moreover, there were age effects within the total sample, justifying age-specific norms. A similar approach is encouraged for creating normative data for people who speak other languages.

\section{DISCLOSURE STATEMENT}

Grant Iverson has received research support from test publishing companies in the past, including PAR, Inc., ImPACT Applications, Inc., and CNS Vital Signs. He receives royalties for one neuropsychological test (Wisconsin Card Sorting Test64 Card Version). He acknowledges unrestricted philanthropic support from the Mooney-Reed Charitable Foundation, ImPACT Applications, Inc., and the Heinz Family Foundation. 


\section{REFERENCES}

Agranovich, A., \& Puente, A. (2007). Do Russian and American normal adults perform similarly on neuropsychological tests? Preliminary findings on the relationship between culture and test performance. Archives of Clinical Neuropsychology, 22, 273-282.

Asken, B. M., McCrea, M. A., Clugston, J. R., Snyder, A. R., Houck, Z. M., \& Bauer, R. M. (2016). "Playing Through It": Delayed Reporting and Removal From Athletic Activity After Concussion Predicts Prolonged Recovery. Journal of Athletic Training, 51, 329-335.

Aubry, M., Cantu, R., Dvorak, J., Johnston, K., Kelly, J., Lovell, M., ... Concussion in Sport Group. (2002). Summary and agreement statement of the first International Conference on Concussion in Sport, Vienna, 2001. British Journal of Sport Medicine, 36, 3-7.

Bure-Reyes, A., Hidalgo-Ruzzante, N., Vilar-Lopez, R., Gontier, J., Sanchez, L., Perez-Garcia, M., \& Puente, A. E. (2013). Neuropsychological test performance of Spanish speakers: Is performance different across different Spanish-speaking groups? Journal of Clinical and Experimental Neuropsychology, 35, 404-412.

Broglio, S. P., Macciocchi, S. N., \& Ferrara, M. S. (2007). Neurocognitive performance of concussed athletes when symptom free. Journal of Athletic Training, 42, $504-508$.

Brooks, B. L., McKay, C. D., Mrazik, M., Barlow, K. M., Meeuwisse, W. H., \& Emery, C. A. (2013). Subjective, but not Objective, Lingering Effects 
of Multiple Past Concussions in Adolescents. Journal of Neurotrauma, 30, 14691475

Brooks, B. L., Mannix, R., Maxwell, B., Zafonte, R., Berkner, P. D.\& Iverson, G. (2016). L. Multiple Past Concussions in High School Football Players: Are There Differences in Cognitive Functioning and Symptom Reporting? American Journal of Sports Medicine. 44, 3243-3251.

Brooks, B. L, Silverberg, N., Maxwell, B., Mannix, R., Zafonte, R., Berkner, B. D. \& Iverson, G. L. Investigating Effects of Sex Differences and Prior Concussions on Symptom Reporting and Cognition Among Adolescent Soccer Players. (2018). American Journal of Sports Medicine. 46, 961-968.

Bruce, J., Echemendia, R., Meeuwisse, W., Comper, P., \& Sisco, A. (2014). 1-year test-retest reliability of ImPACT in professional ice hockey players. The Clinical Neuropsychologist, 28, 14-25.

Cherner, M., Suarez, P., Lazzaretto, D., Fortuny, L. A., Mindt, M. R., Dawes, S., ... the HNRC group. (2007). Demographically corrected norms for the Brief Visuospatial Memory Test-revised and Hopkins Verbal Learning Testrevised in monolingual Spanish speakers from the U.S.-Mexico border region. Archives of Clinical Neuropsychology, 22, 343-353.

Cohen, J. (1988). Statistical power analysis for the behavioral sciences (2nd ed.). Hillsdale, NJ: Erlbaum.

Collins, M. W., Grindel, S. H., Lovell, M. R., Dede, D. E., Moser, D. J., Phalin, B. R., ... McKeag, D. B. (1999). Relationship between concussion and neuropsychological performance in college football players. Journal of the American Medical Association, 282, 964-970. 
Daneshvar, D. H., Nowinski, C. J., McKee, A., \& Cantu, R. C. (2011). The Epidemiology of Sport-Related Concussion. Clinics in Sports Medicine, 30, $1-17$.

Echemendia, R. J, Bruce, J. M, Meeuwisse, W, Comper, P, Aubry, M., \& Hutchison, M. (2016). Long-term reliability of ImPACT in professional ice hockey. The Clinical Neuropsychologist, 30, 328-37.

Echemendia, R. J., Iverson, G. L., McCrea, M., Macciocch, S., Gioia, G., Putukian, M., \& Comper, P. (2013). Advances in neuropsychological assessment of sport-related concussion. British Journal of Sports Medicine, 47, 294-298.

Elbin, R.J., Schatz, P., \& Covassin, T. (2011). One-year test-retest reliability of the online version of ImPACT in high school athletes. American Journal of Sports Medicine 39, 2319-2324.

Elbin, R.J., Sufrinko, A., Schatz, P., French, J., Henry, L., Burkhart, S., Collins, M.W., \& Kontos, A.P. (2016). Removal From Play After Concussion and Recovery Time. Pediatrics. 138, e20160910.

Elbin, R. J., Kontos, A. P., Kegel, N., Johnson, E., Burkhart, S. \& Schatz, P. (2013). Individual and Combined Effects of LD and ADHD on Computerized Neurocognitive Concussion Test Performance: Evidence for Separate Norms. Archives of Clinical Neuropsychology, 28, 476-484.

Farnsworth, J. L., Dargo, L., Ragan, B. G., Kang, M. (2017) Reliability of computerized neurocognitive tests for concussion assessment: A meta-analysis. Journal of Athletic Training, 52, 826-833.

Fazio, V. C., Lovell, M. R., Pardini, J. E., \& Collins, M. W. (2007). The relation between post concussion symptoms and neurocognitive performance in concussed athletes. NeuroRehabilitation, 22, 207-216. 
Grindel, S. H., Lovell, M. R., \& Collins, M. W. (2001). The assessment of sport-related concussion: the evidence behind neuropsychological testing and management. Clinical Journal of Sport Medicine, 11, 134-143.

Hootman, J. M., Dick, R., \& Agel, J. (2007). Epidemiology of Collegiate Injuries for 15 Sports: Summary and Recommendations for Injury Prevention Initiatives. Journal of Athletic Training, 42, 311-319.

Iverson, G. L., Lovell, M. R., \& Collins, M. W. (2003). Interpreting Change on ImPACT Following Sport Concussion. The Clinical Neuropsychologist, 17, 460-467.

Iverson, G. L., Brooks, B. L., Lovell, M. R., \& Collins, M. W. (2006). No cumulative effects for one or two previous concussions. British Journal of Sports Medicine, 40, 72-75.

Langlois, J., Rutland-Brown, W., \& Wald, M. (2006). The Epidemiology and Impact of Traumatic Bain Injury. A Brief Overview. Journal of Head Trauma and Rehabilitation, 21, 375-78.

Lovell, M. R., \& Collins, M. W. (2002). New developments in the evaluation of sports-related concussion. Current Sports Medicine Reports, 1, 287292.

McCrea, M,. Guskiewicz, K., Randolph, C., Barr, W. B., Hammeke, T. A., Marshall, S. W., Kelly, J. P. (2009). Effects of a symptom-free waiting period on clinical outcome and risk of reinjury after sport-related concussion. Neurosurgery, 65, 876-882.

McCrory, P., Meeuwisse, W., Dvorak, J., Aubry, M., Bailes, J., Broglio, S.,... Vos PE. (2017). Consensus statement on concussion in sport-the 5th 
international conference on concussion in sport held in Berlin, October 2016. Journal of Sports Medicine, 51, 838-847.

McKay, C. D., Brooks, B. L., Mrazik, M., Jubinville, A. L., \& Emery, C. (2014). Psychometric Properties and Reference Values for the ImPACT Neurocognitive Test Battery in a Sample of Elite Youth Ice Hockey Players. Archives of Clinical Neuropsychology, 29, 141-151.

Merritt, V. C, Meyer, J. E, Cadden, M. H., Roman, C. A., Ukueberuwa, D. M., Shapiro, M. D., Arnett, P. A. (2017). Normative Data for a Comprehensive Neuropsychological Test Battery used in the Assessment of Sports-Related Concussion. Archives of Clinical Neuropsychology, 32, 168-183.

Norman, M., Moore, D., Taylor, M., Franklin, D., Cysique, L., Ake, C., ... the HNRC Group. (2011). Demographically corrected norms for African Americans and Caucasians on the Hopkins verbal learning test-revised, brief visuospatial memory test-revised, Stroop color and word test, and Wisconsin card sorting test 64-card version. Journal of Clinical and Experimental Neuropsychology, 33, 793-804.

Ott, S., Schatz, P., Solomon, G., \& Ryan, J. (2014). Neurocognitive Performance and Symptom Profiles of Spanish-Speaking Hispanic Athletes on the ImPACT Test. Archives of Clinical Neuropsychology, 29, 152-163.

Peltonen, K., Vartiainen, M., Laitala-Leinonen, T., Koskinen, S., Luoto, T., Pertab, J., \& Hokkanen, L. (2018), Adolescent athletes with learning disability display atypical maturational trajectories on concussion baseline testing: Implications based on a Finnish sample. Child Neuropsychology, 21, 1-16. doi: 10.1080/09297049.2018.1474865. [Epub ahead of print] 
Putukian, M. (2011). Neuropsychological Testing as It Relates to Recovery From Sports-related Concussion. Physical Medicine and Rehabilitation $3,425-432$

Schatz, P., Pardini, J. E., Lovell, M. R., Collins, M. W., \& Podell, K. (2006). Sensitivity and specificity of the ImPACT Test Battery for concussion in athletes. Archives of Clinical Neuropsychology, 21, 91-99.

Schatz, P., \& Robertshaw, S. (2014). Comparing post-concussive neurocognitive test data to normative data presents risks for under-classifying "above average" athletes. Archives of Clinical Neuropsychology, 29, 625-32.

Schmidt, J.D., Register-Mihalik, J.K., Mihalik, J.P., Kerr, Z.Y., \& Guskiewicz, K.M. (2012). Identifying Impairments after concussion: normative data versus individualized baselines. Medicine \& Science in Sports \& Exercise, $44,1621-1628$.

Shuttleworth-Edwards, A. B., Kemp, R. D., Rust, A. L., Muirhead, J. G. L., Hartman, N. P., \& Radloff S. E. (2004). Cross-cultural Effects on IQ Test Performance: A Review and Preliminary Normative Indications on WAIS-III Test Performance. Journal of Clinical and Experimental Neuropsychology, 26, 903-920.

Shuttleworth-Edwards, A., Whitefield-Alexander, V. J., Radloff, S. E., Taylor, A. M., \& Lovell, M. R. (2009). Computerized neuropsychological profiles of South African versus US athletes: A basis for commentary on crosscultural norming issues in the sports concussion Arena. The Physician and Sportsmedicine, 37, 45-52.

Tsushima, W. T., \& Siu, A. M. (2014). Neuropsychological Test Performance of Hawai'i High School Athletes: Updated Hawai'i Immediate Post- 
Concussion Assessment and Cognitive Testing Data. Hawai' $i$ Journal of Medicine \& Public Health, 73, 208-211.

Vartiainen, V. M., Holm, A., Peltonen, K., Luoto, T., Iverson, G. L., \& Hokkanen, L. (2015). King-Devick test normative reference values for professional players. Scandinavian Journal of Medicine \& Science in Sports, 25, e327-e330

Zakzanis, K.K. (2001). Statistics to tell the truth, the whole truth and nothing but the truth: Formulae, illustrative numerical examples and heuristic interpretation of effect size analyses for neuropsychological researchers. Archives of Clinical Neuropsychology, 16, 653-667.

Zuckerman, S. L., Lee, Y. M., Odom, M. J., Solomon, G. S. \& Sills, A. K. (2013). Baseline neurocognitive scores in athletes with attention deficitspectrum disorder and /or learning disability. Journal of Neurosurgery: Pediatrics, 12, 103-109 
Table 1. Comparisons of ImPACT® test results for professional ice hockey players across language groups.

\begin{tabular}{|c|c|c|c|c|c|c|c|c|c|c|c|c|c|}
\hline \multirow[t]{2}{*}{ Total Sample } & \multirow[b]{2}{*}{$\mathrm{n}$} & \multicolumn{2}{|c|}{ Age } & \multicolumn{2}{|c|}{ Education } & \multicolumn{2}{|c|}{ Verbal Memory } & \multicolumn{2}{|c|}{ Visual Memory } & \multicolumn{2}{|c|}{ Visual Motor Speed } & \multicolumn{2}{|c|}{ Reaction Time } \\
\hline & & $M$ & $S D$ & $M$ & $S D$ & $M$ & $S D$ & $M$ & $S D$ & $M$ & $S D$ & $M$ & $S D$ \\
\hline Finnish & 184 & 23.8 & 5.6 & 11.58 & 1.4 & 85.35 & 9.27 & 75.43 & 12.12 & 39.10 & 6.27 & 0.61 & 0.09 \\
\hline $\mathrm{Brt}$ & & & & & & & & & & & & & \\
\hline English speaking & 119 & 25.49 & 4.61 & 12.72 & 1.77 & 87.92 & 8.39 & 77.81 & 11.92 & $41.42 * *$ & 5.83 & $0.57 * * *$ & 0.07 \\
\hline French speaking & 43 & 24.60 & 4.99 & 11.40 & 1.77 & 84.63 & 8.10 & 78.60 & 11.81 & 39.03 & 6.04 & 0.58 & 0.08 \\
\hline Czech speaking & 42 & 25.67 & 5.36 & 14.91 & 2.84 & 82.38 & 9.74 & 71.76 & 14.05 & $35.51 * *$ & 8.51 & 0.65 & 0.10 \\
\hline Swedish speaking & 28 & 26.62 & 5.23 & 11.69 & 1.14 & 87.96 & 5.97 & 76.46 & 12.03 & 39.71 & 5.12 & 0.56 & 0.07 \\
\hline
\end{tabular}

Note: Higher scores on Reaction Time reflect poor performance (i.e., slower reaction time). $\mathrm{n}=$ Sample size, $M=$ Mean, $S D=$ Standard deviation $* *<0.01$ pairwise comparison to the Finnish sample, significant also after Bonferroni correction

$* * *<0.001$ pairwise comparison to the Finnish sample, significant also after Bonferroni correction 
Table 2. Characteristics of athletes in the three age groups.

\begin{tabular}{|c|c|c|c|c|}
\hline & $\begin{array}{l}\text { Age }<20 \\
\mathrm{n}=53\end{array}$ & $\begin{array}{l}\text { Age } 20-26 \\
\mathrm{n}=72\end{array}$ & $\begin{array}{l}\text { Age }>26 \\
\mathrm{n}=59\end{array}$ & Chi-square, $p$ \\
\hline $\begin{array}{r}\text { Education level } \\
\text { Below } 12 \text { years } \\
12 \text { years or above } \\
\end{array}$ & $\begin{array}{c}48(94 \%) \\
3(6 \%)\end{array}$ & $\begin{array}{l}35(52 \%) \\
32(48 \%)\end{array}$ & $\begin{array}{l}19(35 \%) \\
35(65 \%)\end{array}$ & $40.0 * * *$ \\
\hline $\begin{array}{r}\text { Concussion history } \\
1 \text { None } \\
1 \text { or more } \\
\end{array}$ & $\begin{array}{l}41(77 \%) \\
12(23 \%)\end{array}$ & $\begin{array}{l}35(49 \%) \\
37(51 \%)\end{array}$ & $\begin{array}{l}23(39 \%) \\
36(61 \%)\end{array}$ & $17.8 * * *$ \\
\hline Special education needs & $\begin{array}{r}47(89 \%) \\
6(11 \%)\end{array}$ & $\begin{array}{r}64(89 \%) \\
8(11 \%)\end{array}$ & $\begin{array}{c}54(91 \%) \\
5(9 \%)\end{array}$ & 0.32 \\
\hline
\end{tabular}


Table 3. Finnish reference values for ImPACT® Composite Scores

\begin{tabular}{|c|c|c|c|c|c|c|c|c|c|c|}
\hline & $n$ & Mean & Median & $S D$ & Superior & $\begin{array}{l}\text { Above } \\
\text { Average }\end{array}$ & Average & $\begin{array}{l}\text { Below } \\
\text { Average }\end{array}$ & $\begin{array}{c}\text { Well Below } \\
\text { Average }\end{array}$ & $\begin{array}{l}\text { Extremely } \\
\text { Low }\end{array}$ \\
\hline Percentile & & & & & $>90^{\text {th }}$ & $76^{\text {th }}-90^{\text {th }}$ & $25^{\text {th }}-75^{\text {th }}$ & $10^{\text {th }}-24^{\text {th }}$ & $2^{\text {nd }}-9^{\text {th }}$ & $<2^{\text {nd }}$ \\
\hline \multicolumn{11}{|l|}{ Age $<20$} \\
\hline Verbal Memory & 53 & 83.98 & 85.00 & 10.07 & $>98.20$ & $98.20-90.51$ & $90.50-76.50$ & $76.51-71.20$ & $71.19-55.00$ & $<55.00$ \\
\hline Visual Memory & 53 & 74.94 & 75.00 & 12.58 & $>91.00$ & $91.00-83.51$ & $84.50-66.00$ & $66.01-57.80$ & $57.79-48.00$ & $<48.00$ \\
\hline $\begin{array}{l}\text { Visual Motor } \\
\text { Speed }\end{array}$ & 53 & 39.32 & 39.75 & 5.89 & $>46.64$ & $46.64-42.84$ & $42.83-34.90$ & $34.89-31.53$ & $31.54-21.97$ & $<21.97$ \\
\hline Reaction Time & 53 & 0.59 & 0.57 & 0.08 & $<0.51$ & $0.51-0.52$ & $0.53-0.63$ & $0.64-0.66$ & $0.67-0.88$ & $>0.88$ \\
\hline Impulse Control & 53 & 5.36 & 4.0 & 4.26 & $<1.40$ & $1.40-2.99$ & $3.00-7.00$ & $7.01-10.20$ & $10.21-20.00$ & $>20.00$ \\
\hline & & & & & & & & & & \\
\hline \multicolumn{11}{|l|}{ Age $20-26$} \\
\hline Verbal Memory & 72 & 86.39 & 88.00 & 8.26 & $>96.00$ & $96.00-93.00$ & $93.01-81.00$ & $81.01-74.00$ & $74.01-66.38$ & $<66.38$ \\
\hline Visual Memory & 72 & 75.96 & 77.50 & 11.40 & $>91.70$ & $91.70-82.00$ & $82.01-69.00$ & $69.01-59.30$ & $59.31-50.84$ & $<50.84$ \\
\hline $\begin{array}{l}\text { Visual Motor } \\
\text { Speed }\end{array}$ & 72 & 40.23 & 41.21 & 6.03 & $>48.93$ & $48.93-44.00$ & $44.01-36.01$ & $36.02-32.06$ & $32.07-27.04$ & $<27.04$ \\
\hline Reaction Time & 72 & 0.59 & 0.59 & 0.08 & $<0.49$ & $0.49-0.53$ & $0.54-0.65$ & $0.66-0.72$ & $0.73-0.76$ & $>0.76$ \\
\hline Impulse Control & 72 & 4.60 & 4.00 & 3.18 & $<1.00$ & $1.00-2.00$ & $2.01-6.00$ & $6.01-8.00$ & $8.01-15.16$ & $>15.16$ \\
\hline \multicolumn{11}{|l|}{ Age $>26$} \\
\hline Verbal Memory & 59 & 85.32 & 85.00 & 9.69 & $>99.00$ & $99.00-93.00$ & $93.01-78.00$ & $78.01-73.00$ & $73.01-61.60$ & $<61.60$ \\
\hline Visual Memory & 59 & 75.22 & 75.00 & 12.73 & $>92.00$ & $92.00-84.00$ & $84.01-66.00$ & $66.01-58.00$ & $58.01-38.60$ & $<38.60$ \\
\hline $\begin{array}{l}\text { Visual Motor } \\
\text { Speed }\end{array}$ & 59 & 37.50 & 36.28 & 6.66 & $>47.90$ & $47.90-42.83$ & $42.84-33.67$ & $33.68-30.03$ & $30.04-19.64$ & $<19.64$ \\
\hline Reaction Time & 59 & 0.65 & 0.62 & 0.11 & $<0.55$ & $0.55-0.60$ & $0.61-0.66$ & $0.67-0.81$ & $0.82-1.14$ & $>1.14$ \\
\hline Impulse Control & 59 & 2.51 & 2.00 & 1.81 & $<0.00$ & $0.00-1.00$ & $1.01-4.00$ & $4.01-5.00$ & $5.01-8.40$ & $>8.40$ \\
\hline
\end{tabular}

$n=$ sample size, $S D=$ standard deviation

Note: Superior scores occur in fewer than $10 \%$ of the sample. Above average scores occur in approximately $15 \%$, average scores occur in approximately

$50 \%$, below average scores occur in approximately $15 \%$, well below average scores occur in approximately $8 \%$, and extremely low scores occur in fewer than $2 \%$. For Reaction Time and Impulse Control, higher scores reflect poor performance (i.e., slower reaction time and more errors). In other composite scores, higher scores reflect better performance. 